\title{
Combination of calcium sulfate and simvastatin- controlled release microspheres enhances bone repair in critical-sized rat calvarial bone defects
}

This article was published in the following Dove Press journal:

International Journal of Nanomedicine

I December 2015

Number of times this article has been viewed

\author{
Yin-Chih Fu ${ }^{1-4}$ \\ Yan-Hsiung Wang ${ }^{1,5}$ \\ Chung-Hwan Chen 1,3,4 \\ Chih-Kuang Wang',6 \\ Gwo-Jaw Wang ${ }^{1,3,4}$ \\ Mei-Ling $\mathrm{Ho}^{1,3,7,8}$ \\ 'Orthopaedic Research Center, \\ ${ }^{2} \mathrm{Graduate}$ Institute of Medicine, \\ ${ }^{3}$ Department of Orthopaedics, \\ ${ }^{4}$ Department of Orthopaedics, \\ College of Medicine, ${ }^{5}$ School of \\ Dentistry, College of Dental Medicine, \\ ${ }^{6}$ Department of Medicinal and Applied \\ Chemistry, ${ }^{7}$ Department of Physiology, \\ College of Medicine, Kaohsiung \\ Medical University, Kaohsiung, Taiwan; \\ ${ }^{8}$ Department of Marine Biotechnology \\ and Resources, National Sun Yat-sen \\ University, Kaohsiung, Taiwan
}

Correspondence: Mei-Ling Ho Department of Physiology, College of Medicine, Kaohsiung Medical University, 100 Shih-Chuan Ist Road, Kaohsiung 807, Taiwan

Email homelin@cc.kmu.edu.tw

\begin{abstract}
Most allogenic bone graft substitutes have only osteoconductive properties. Developing new strategies to improve the osteoinductive activity of bone graft substitutes is both critical and practical for clinical application. Previously, we developed novel simvastatinencapsulating poly(lactic-co-glycolic acid) microspheres (SIM/PLGA) that slowly release simvastatin and enhance fracture healing. In this study, we combined SIM/PLGA with a rapidly absorbable calcium sulfate (CS) bone substitute and studied the effect on bone healing in critical-sized calvarial bone defects in a rat model. The cytotoxicity and cytocompatibility of this combination was tested in vitro using lactate dehydrogenase leakage and a cell attachment assay, respectively. Combination treatment with SIM/PLGA and the CS bone substitute had no cytotoxic effect on bone marrow stem cells. Compared with the control, cell adhesion was substantially enhanced following combination treatment with SIM/PLGA and the CS bone substitute. In vivo, implantation of the combination bone substitute promoted healing of criticalsized calvarial bone defects in rats; furthermore, production of bone morphogenetic protein-2 and neovascularization were enhanced in the area of the defect. In summary, the combination of SIM/PLGA and a CS bone substitute has osteoconductive and osteoinductive properties, indicating that it could be used for regeneration of bone in the clinical setting.
\end{abstract}

Keywords: calcium sulfate, simvastatin, calvarial bone defects, osteoconductive, osteoinductive

\section{Introduction}

Previous studies have estimated that 7.9 million fractures occur each year in the USA, and 5\%-20\% of all fractures result in delayed union or non-union. ${ }^{1-4}$ Approximately 500,000 bone grafting procedures are performed annually in the USA, and 2.2 million are performed worldwide in orthopedics, neurosurgery, and dentistry. ${ }^{5,6}$ The classic standard treatment for bone grafting is the autologous bone graft, which has both osteoconductive and osteoinductive properties. However, autograft harvesting is typically associated with an $8.5 \%-20 \%$ risk of complications, including poor cosmesis, infection, and chronic pain at the donor site. ${ }^{7-9}$ Allografts, which are easily obtained and managed, are effective alternatives for avoiding the disadvantages of autografts, but carry a risk of transmission of live viral disease and bacterial infection. Therefore, a variety of bone substitutes has been introduced.

An ideal bone graft substitute should be osteoconductive, osteoinductive, biocompatible, bioresorbable, and easy to use. ${ }^{10,11}$ Bone graft alternatives are commercially available for orthopedic use, but most are osteoconductive and have little or no osteoinductive capability. Therefore, encapsulating osteoinductive proteins or drugs 
in bone substitutes with controlled-release properties is the major challenge.

Calcium sulfate (CS) has been used as a bone void filler since the late 1980s. It has resorbable osteoconductive properties but may not provide sufficient biomechanical support and osteoconductivity in large bone defects because it generally dissolves within 6-12 weeks. ${ }^{10}$ Therefore, it is not recommended for non-unions or periarticular fractures. However, because of its resorbability, CS can be used as an implant device when loaded with certain materials (eg, antibiotics, pharmacological agents, growth factors) without any evidence of local or systemic adverse effects. ${ }^{11-14}$

Calcium phosphate (CP) ceramics have been used since the $1980 \mathrm{~s}$ in orthopedics, ${ }^{15}$ and are similar in composition to an inorganic apatite. ${ }^{16,17}$ In general, CP and hydroxyapatite (HAp) show effective biocompatibility, with osteoconductive properties and without immunogenic or toxic side effects. ${ }^{16,17}$ However, HAp-sintered ceramics, which are widely used, have limited bioresorbability, causing the HAp to remain in the body long after implantation. ${ }^{18} \mathrm{CP}$ exhibits superior degradation characteristics during bone regeneration compared with HAp. ${ }^{19}$ However, the degradation is extremely slow in non-loaded critical-sized defects. ${ }^{20}$ Therefore, Passuti et al have modified and combined other materials (HAp or CP alone or combined with autogenous bone grafts) to improve functionality and promote rapid resorption. ${ }^{21}$ The limitations of these materials are that ceramics are available only as powders, cements, or porous implants, and have a number of disadvantages such as difficulty in implantation, a long duration required for complete integration and replacement with newly formed bone, and an inability to fill irregular gaps. Therefore, they are not useful when used alone in the treatment of atrophic nonunions of long bones because of the lack of growth factors and vascularity. ${ }^{22}$

Bone morphogenetic proteins (BMPs) are low-molecularweight glycoproteins with pleomorphic functions ranging from extracellular and skeletal organogenesis to bone generation and regeneration. ${ }^{23}$ Generally, the clinical application of native growth factors without carriers is limited by their short half-life and the problem of bursting release. ${ }^{23}$ The Infuse $^{\circledR}$ bone graft (recombinant human BMP-2 combined with collagen fleece, Medtronic) has been approved by the US Food and Drug Administration as an autograft replacement material for certain indications, excluding use in cervical spine fusion. ${ }^{24-26}$ Therefore, identification of non-protein small molecules to stimulate fracture healing would provide an alternative method.
Simvastatin, a 3-hydroxy-3-methylglutaryl coenzyme A reductase inhibitor, is used clinically to reduce blood cholesterol levels. ${ }^{27,28}$ Mundy et al demonstrated that statins stimulate BMP-2 gene expression in osteoblasts. ${ }^{29}$ Our previous research has shown that the controlled-release properties of simvastatin microspheres not only enhance callus formation during initiation of fracture healing but also increase neovascularization and cell growth in the grafted bone. ${ }^{30}$

The objective of this study was to identify a bioactive bone substitute that induces tissue regeneration and enhances critical bone defect healing. We hypothesized that the combined use of controlled-release simvastatin-encapsulating poly(lactic-co-glycolic acid) microspheres (SIM/PLGA) and rapidly absorbable CS with drug-carrying properties producing osteoinductive and osteoconductive cues can enhance bone healing in critical bone defects. We evaluated the cytocompatibility of this combination in cultured bone marrow mesenchymal stem (D1) cells and assessed its osteogenic effect using a rat model of critical calvarial defects.

\section{Materials and methods Cell culture}

We purchased the mouse bone marrow mesenchymal stem cells from the American Type Culture Collection, maintained them in Dulbecco's Modified Eagle's Medium (Gibco BRL, Life Technologies, Invitrogen), and supplemented them with $10 \%$ fetal bovine serum (BD Biosciences), 0.15\% sodium bicarbonate (Sigma-Aldrich), $10 \mathrm{mM}$ nonessential amino acids (Gibco), 0.01\% vitamin C (Gibco), and $100 \mathrm{U} / \mathrm{mL}$ of penicillin/streptomycin (Gibco) in a humidified 5\% $\mathrm{CO}_{2}$ atmosphere at $37^{\circ} \mathrm{C}$. The medium was changed every 2 days.

\section{Lactate dehydrogenase assay}

The cytotoxicity of the bone graft substitute was analyzed using an indirect method according to the instructions of ISO10993-5. The sterilized bone graft substitute was incubated with culture medium at $37^{\circ} \mathrm{C}$ for 24 hours. Subsequently, the extraction medium was collected for analysis of cytotoxicity. D1 cells were seeded into 96-well culture plates at a density of $1 \times 10^{4}$ cells per well 1 day before the lactate dehydrogenase (LDH) assay. Next, the culture medium was replaced with the extraction medium obtained from the bone graft substitute and cultured for 24 hours. Cytotoxicity was evaluated by detecting LDH activity using an LDH assay kit (Clontech) according to the manufacturer's instructions. A medium with $10 \%$ phenol (Sigma-Aldrich) was used as 
the positive control, and the culture medium was used as the negative control.

\section{Cell attachment assays}

For cell attachment tests, D1 cells were seeded on sterilized bone grafts at a density of $1 \times 10^{5}$ cells $/ \mathrm{mL}$. Three hours after the cells became adhered to the bone grafts, the cell/bone graft constructs were rinsed and removed from the 48-well cell culture plates. The number of unattached viable cells was counted using a handheld Scepter automated cell counter (Millipore). Cells seeded on 48-well culture plates without bone grafts were used as the control, and a polyvinyl chloride disk was used as the negative control.

\section{Fabrication of CS-based bone graft substitutes}

Three milligrams of PLGA or SIM/PLGA (sustained-release for 14 days) $)^{30}$ were tableted to $5 \mathrm{~mm}$ in diameter to fit the critical size of the defect. The commercial artificial bone substitute Osteoset ${ }^{\circledR}$ (medical grade CS pellets, Wright Medical Group) was used in this study. The bone graft substitutes were fabricated according to the manufacturer's instructions, and $32 \mathrm{mg}$ of Osteoset was used. Subsequently, PLGA or SIM/PLGA and CS pellets were compressed in disc-shaped Teflon molds to form discs that were $0.5 \mathrm{~mm}$ thick, and were dried at room temperature.

\section{Animals}

A total of 60 male Sprague-Dawley rats (age 8 weeks, weight 200-350 g) were used in this study. All animals were housed at the Kaohsiung Medical University Animal Research Center, and the experimental procedures were approved by the Institutional Animal Care and Use Committee (Kaohsiung Medical University approval 98186). All rats with calvarial defects were randomized into four groups (15 rats/group) as follows: a control (CS) group in which the calvarial defect was treated with an Osteoset bone graft alone; a CS/PLGA group treated with Osteoset + PLGA microspheres; and a CS/SIM/PLGA group treated with Osteoset and SIM/PLGA. The rats were euthanized at 8,10 , or 12 weeks post implantation, after which the calvarial specimens were harvested, fixed in $4 \%$ paraformaldehyde at $4^{\circ} \mathrm{C}$ for 24 hours, and subsequently analyzed for histomorphology.

\section{Critical-sized calvarial defects in rats}

The critical-sized calvarial defects $(5 \mathrm{~mm}$ in width and approximately $0.7 \mathrm{~mm}$ in depth) were prepared according to our previous study. ${ }^{31}$ The bone graft substitute constructs were implanted under anesthesia by intraperitoneal injection of ketamine (Parke-Davis; $10 \mathrm{mg} / 100 \mathrm{~g}$ body weight) in combination with xylazine hydrochloride (Bayer HealthCare; $12 \mathrm{mg} / 100 \mathrm{~g}$ body weight). The hair over the calvarium was shaved with a depilator and cleaned. A midline calvarial incision was made and a $5 \mathrm{~mm}$ hole was drilled to penetrate through the calvarial bone using a trephine burr with constant phosphate-buffered saline irrigation. Extreme care was taken to avoid damaging the dura mater. Rats with critical-sized $(5 \mathrm{~mm})$ calvarial defects were implanted with or without bone graft substitutes in the defect sites. The wounds were sutured after implantation with 5-0 nylon sutures. All rats survived and showed no wound infections throughout the experiment.

\section{Histological analysis and immunostaining of bone tissue}

Quantitative histochemical and immunohistochemical analyses were used to investigate the microscopic changes in bone tissue. Prior to hematoxylin-eosin and immunohistochemistry staining, all bone tissue samples were decalcified $(0.5 \mathrm{M}$ ethylenediaminetetraacetic acid- $2 \mathrm{H}_{2} \mathrm{O}$ in double-distilled water $[186.1 \mathrm{~g} / \mathrm{L}])$, followed by fixation with $4 \%$ paraformaldehyde. The samples were then embedded in paraffin wax. We divided the samples into frontal and rear portions according to the middle of the defect in each sample. For each side, $5 \mu \mathrm{m}$ sections were prepared sequentially from the mid-line to the edge, and 15 sections were collected from each side. A total of 30 sections were obtained from the front to the rear in each sample. Sections 1-3 were defined as the frontal area, sections 14-16 as the middle area, and sections 28-30 as the rear area. These sections were routinely stained with hematoxylin-eosin. At a magnification of $40 \times$, we counted the area of bone matrix formation in the calvarial defect, and the area was measured using Image-Pro Plus 5.0 software (Media Cybernetics Inc). The percentage of bone matrix within the calvarial defect was calculated and compared with that of the control group. Immunohistochemistry staining for BMP-2 and von Willebrand factor (vWF) was performed. The sections were treated with $1 \mathrm{mg} / \mathrm{mL}$ pronase (SigmaAldrich) for 1 hour and then incubated overnight at $4{ }^{\circ} \mathrm{C}$ with a 1:700 dilution of polyclonal rabbit anti-vWF antibody (Millipore). Goat anti-rabbit immunoglobulin (Abcam) was used in a 1:2,000 dilution as the secondary antibody at $37^{\circ} \mathrm{C}$ for 60 minutes. Peroxidase activity was detected using $0.4 \mathrm{mg} / \mathrm{L}$ of 3,3-diaminobenzidine in phosphate buffer at pH 7.3 in the presence of $0.12 \% \mathrm{H}_{2} \mathrm{O}_{2}$. The sections were 
then counterstained with hematoxylin. At a magnification of $400 \times$, the number of stained endothelial vessels within the surgical calvarial defects was calculated and compared between the samples and controls.

\section{Statistical analysis}

Each experiment was repeated at least three times, and the data (expressed as the mean \pm standard error of the mean) from representative experiments are shown. Statistical significance was evaluated by one-way analysis of variance, and multiple comparisons were performed using Scheffe's method. A value of $P<0.05$ was considered to be statistically significant.

\section{Results}

\section{Cytotoxicity of CS combined with SIM/PLGA}

The cytotoxic effects of the bone grafts were measured using the LDH assay. Except for the phenol-treated (positive control) group, the LDH activity exhibited no changes after the D1 cells were cultured with the extraction medium from the bone graft substitutes (Figure 1).

\section{Cell affinity for CS combined with SIM/PLGA}

We tested the bone graft properties of cell attachment using a cell attachment assay. Approximately $77 \%$ and $32 \%$ of the D1 cells were attached on the CS/SIM/PLGA and CS bone grafts, respectively (Figure 2). Only $4.5 \%$ of the D1 cells were attached on the polyvinyl chloride (negative control) material (Figure 2).

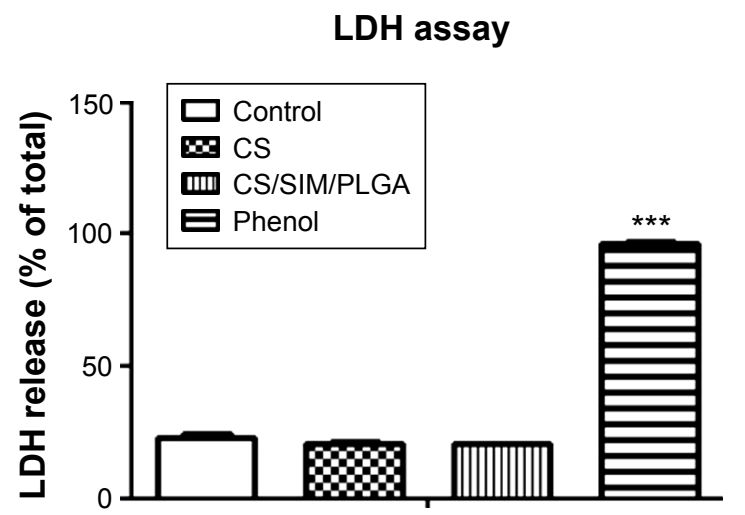

Figure I The cytotoxic effects of Phenol (positive control), CS and CS/SIM/PLGA bone grafts.

Notes: DI cells were treated with extraction medium obtained from the bone grafts. LDH leakage was analyzed to evaluate cell cytotoxicity. The values are shown as the mean \pm standard error of the mean, $n=4$. $* * * P<0.001$ compared with control group. Abbreviations: CS, calcium sulfate; SIM, simvastatin; PLGA, poly(lactic-co-glycolic acid) microspheres; LDH, lactate dehydrogenase.

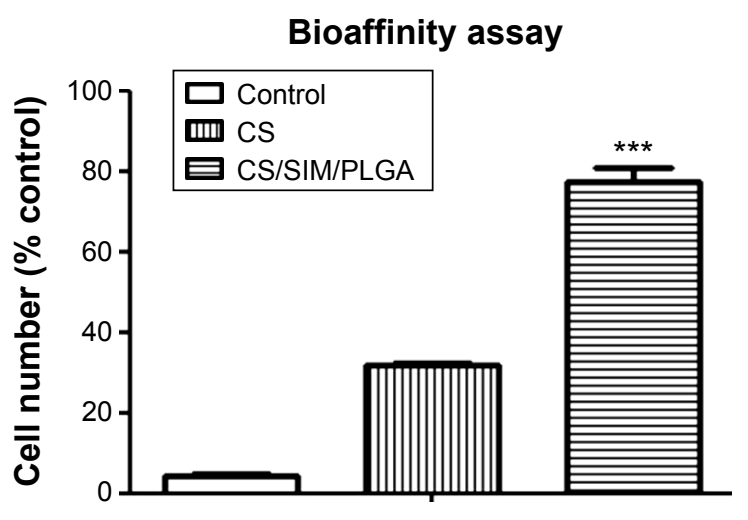

Figure 2 Cell adherent effect of DI cells seeded on PVC (negative control), CS, and CS/SIM/PLGA bone grafts.

Notes: The values are shown as the mean \pm standard error of the mean, $n=3$. *** $P<0.00$ I compared with the PVC group.

Abbreviations: PVC, polyvinyl chloride; CS, calcium sulfate; SIM, simvastatin; PLGA, poly(lactic-co-glycolic acid) microspheres.

\section{Hematoxylin-eosin staining of critical- sized calvarial defects}

The effect of CS with or without SIM/PLGA on bone repair was evaluated at 8,10 , and 12 weeks after surgery (Figures 3-5). The rat calvaria were harvested and analyzed using hematoxylin-eosin staining. The region of the calvarial defects in the control and CS groups revealed mostly fibrous tissue with minimal bone formation at 8 weeks after implantation. The calvarial defects of the CS/PLGA and CS/ SIM/PLGA groups maintained higher matrix-filled areas and cellularity than the control and CS groups (Figures 3-5). At 10 weeks after surgery, only the CS/SIM/PLGA group showed obvious new bone formation in the calvarial defects; the other three groups exhibited patterns similar to those seen at 8 weeks after surgery (Figure 4 ). The bone healing process in all groups at 12 weeks after surgery (Figure 5) was similar to that after 10 weeks of bone healing, as shown in Figure 4. After quantification of new bone formation, the CS/SIM/ PLGA group exhibited substantially increased new bone formation in the critical-sized calvarial defect at 10 and 12 weeks after surgery (Figure 6). In addition, the CS and $\mathrm{CS} / \mathrm{SIM} / \mathrm{PLGA}$ groups showed more new bone formation than the control and CS/PLGA groups at 8 weeks after surgery.

\section{Immunohistochemistry staining of critical-sized calvarial defects}

Immunohistochemistry was used to examine BMP-2 (Figures 7 and 8) and vWF (Figures 9 and 10) expression in newly formed bone at 8,10 , and 12 weeks after surgery and also to investigate whether SIM/PLGA enhanced 


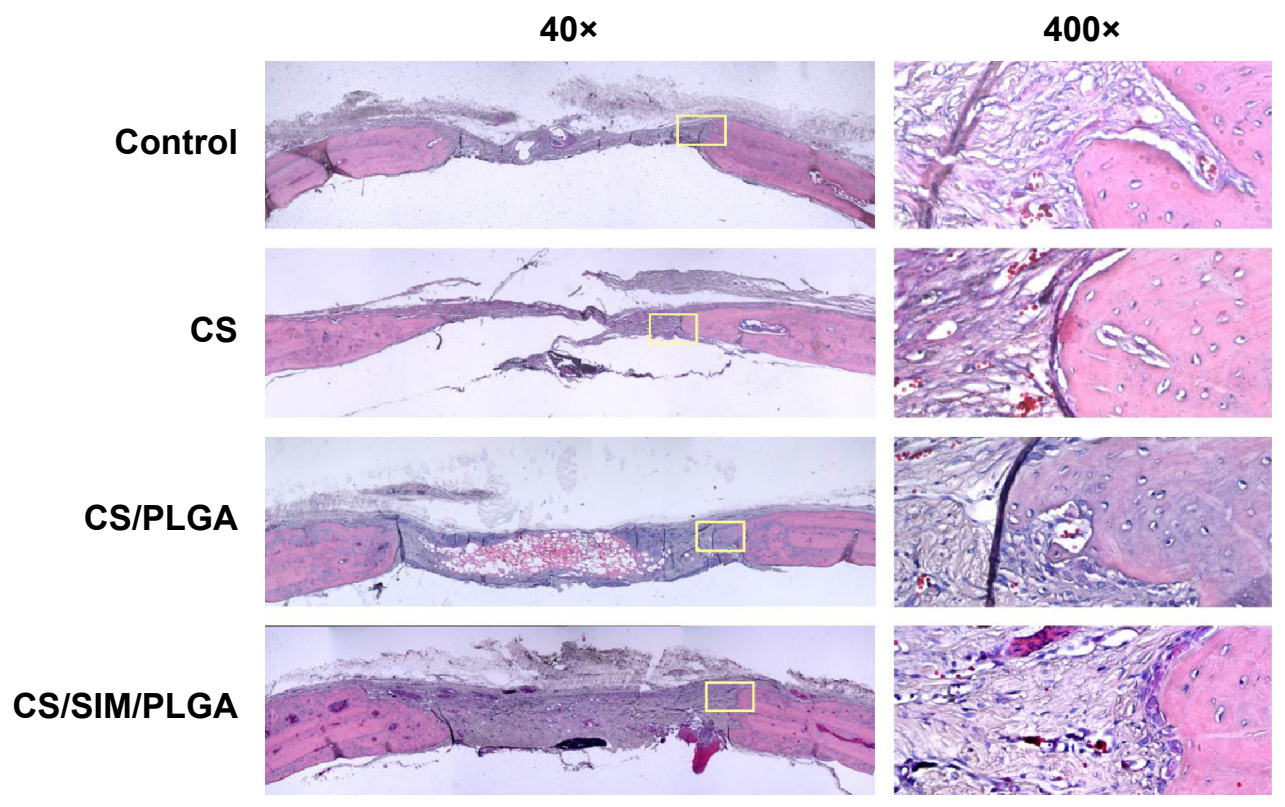

Figure 3 Histological specimens from calvarial defects 8 weeks after implantation of bone graft substitutes with hematoxylin-eosin staining. Note: Calvarial defects implanted without bone graft substitutes were used as controls.

Abbreviations: CS, calcium sulfate; SIM, simvastatin; PLGA, poly(lactic-co-glycolic acid) microspheres.

bone healing by stimulating osteogenesis and neovascularization. No or little BMP-2 expression was observed at the defect area in the control group at all three time points (Figures 7A-C and 8A-C). Minor BMP-2 expression was observed at the defect area in the CS group at 8 and 10 weeks after surgery (Figures 7D-E and 8D-E), but lower
BMP-2 expression was found at 12 weeks after surgery (Figures $7 \mathrm{~F}$ and $8 \mathrm{~F}$ ). In the CS/PLGA group (Figures 7G-I and $8 \mathrm{G}-\mathrm{I}), \mathrm{BMP}-2$ expression was extremely low at 8 weeks after surgery, and minor BMP-2 expression was observed in the defect area at 8 and 10 weeks after surgery. Obvious BMP-2 expression was observed in the CS/SIM/

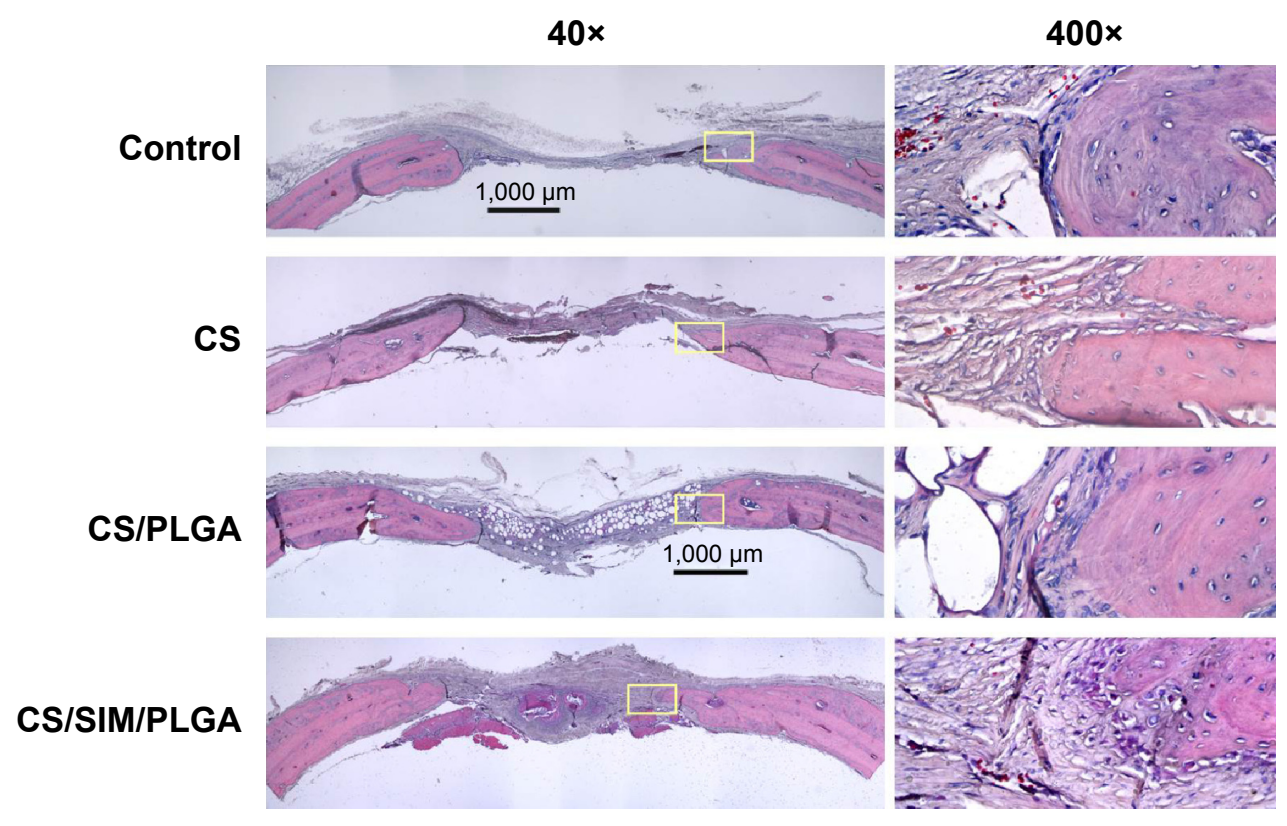

Figure 4 Histological specimens from calvarial defects 10 weeks after implantation of bone graft substitutes with hematoxylin-eosin staining. Note: Calvarial defects implanted without bone graft substitutes were used as controls.

Abbreviations: CS, calcium sulfate; SIM, simvastatin; PLGA, poly(lactic-co-glycolic acid) microspheres. 


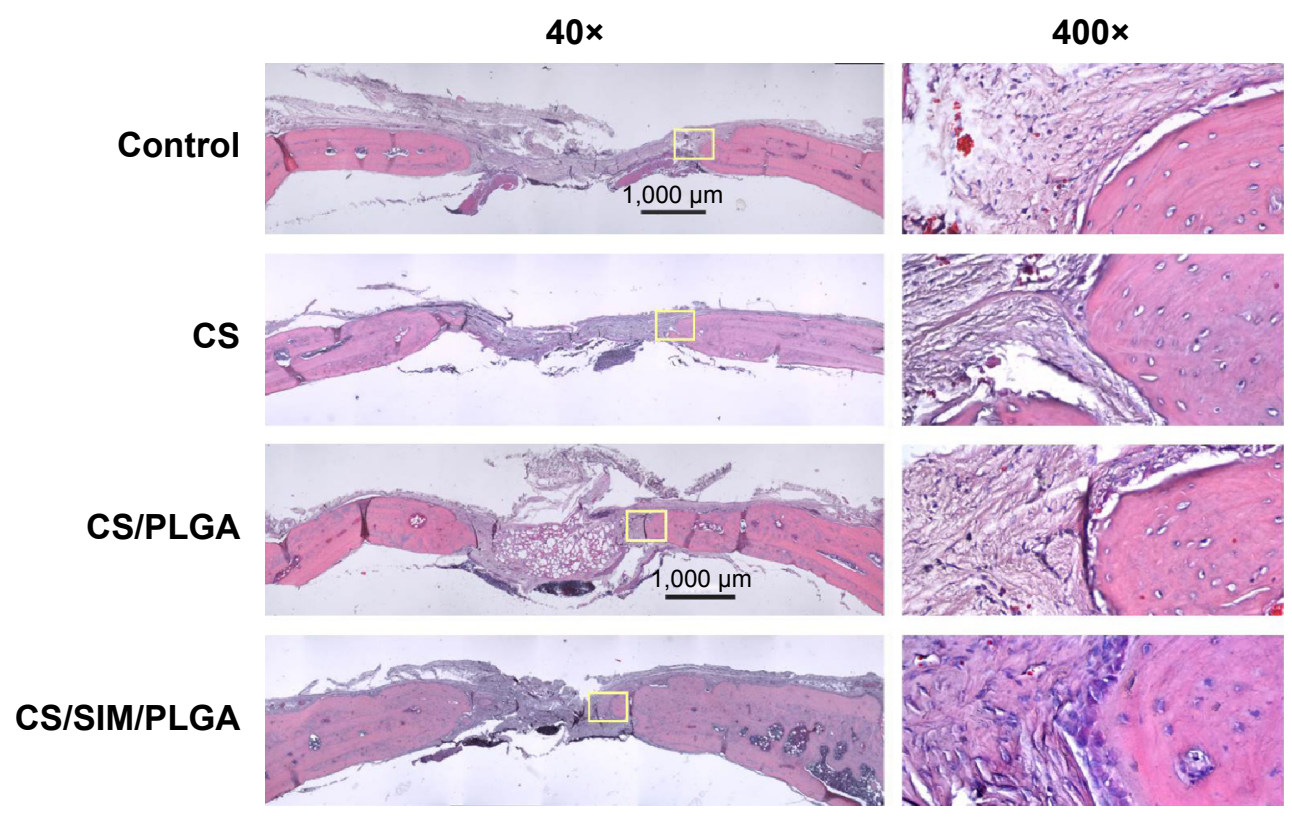

Figure 5 Histological specimens from calvarial defects after 12 weeks of implantation of bone graft substitutes with hematoxylin-eosin staining. Note: Calvarial defects implanted without bone graft substitutes were used as controls.

Abbreviations: CS, calcium sulfate; SIM, simvastatin; PLGA, poly(lactic-co-glycolic acid) microspheres.

PLGA group at 8 weeks after surgery (Figures $7 \mathrm{~J}$ and $8 \mathrm{~J}$ ), and a similar expression pattern continued through weeks 10-12 after surgery (Figures $7 \mathrm{~K}-\mathrm{L}$ and $8 \mathrm{~K}-\mathrm{L}$ ).

With regard to neovascularization, limited vWF expression was observed at the defect area in the control, CS, and CS/PLGA groups at all three time points (Figures 9A-I and 10A-I). However, in the CS/SIM/PLGA group, vWF expression was observed at 8 weeks after surgery and uniformly

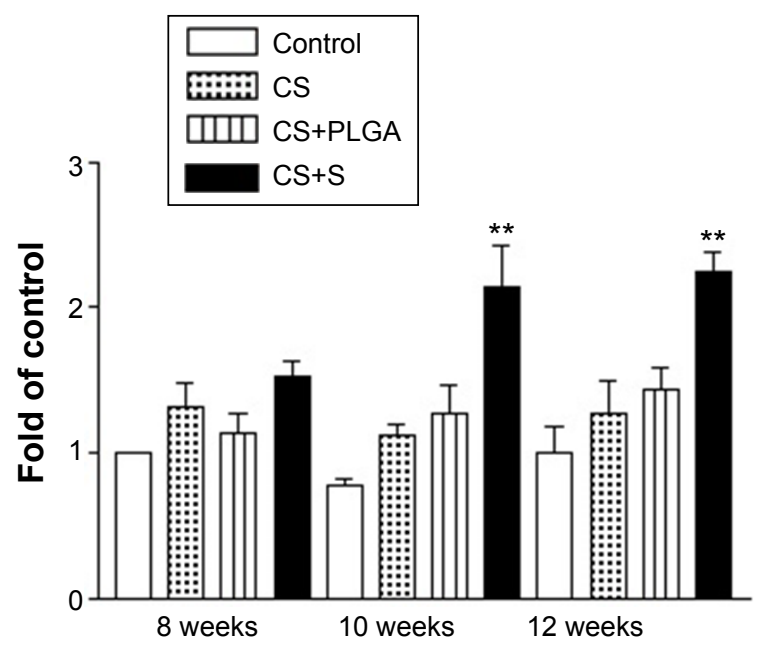

Figure 6 Histological quantification study of new bone matrix formation at 8, 10, and 12 weeks after implantation of bone graft substitutes.

Note: $* * P<0.000$ I compared with controls and between treatment groups, respectively.

Abbreviations: CS, calcium sulfate; SIM, simvastatin; PLGA, poly(lactic-co-glycolic acid) microspheres. distributed in the defect area (Figures 9J and 10J). Obvious vWF expression was observed at weeks 10-12 after surgery (Figures 9K-L and 10K-L).

\section{Discussion}

An optimal bone substitute for critical-sized bone defects should not only be biocompatible and osteoconductive but also requires an osteoinductive environment for bone regeneration. ${ }^{32}$ In this study, we used CS (Osteoset) as a bone graft substitute because of its rapid resorption and ability to carry controlled-release SIM/PLGA for local delivery to enhance bone healing. Before clinical application, safety has to be ensured, and easy attraction of cells attaching to the scaffold surface has to be demonstrated. First, we confirmed that this composition was safe in vitro. Although controlledrelease simvastatin microspheres were shown to have no cytotoxic effect in a previous study, ${ }^{30}$ the use of a CS bone substitute combined with SIM/PLGA required further evaluation to determine whether such a combination is cytotoxic. We confirmed in LDH assay that CS/SIM/PLGA did not result in any cytotoxic effects. In addition, we showed that D1 cells attached to the bone substitute complex (CS/SIM/ PLGA) using an attachment test. This indicates that the CS/ SIM/PLGA bone substitute was not cytotoxic and exhibited high cellular affinity. We expect that this new bone substitute would attract bone marrow stem cells and provide an appropriate niche for cells to grow. 


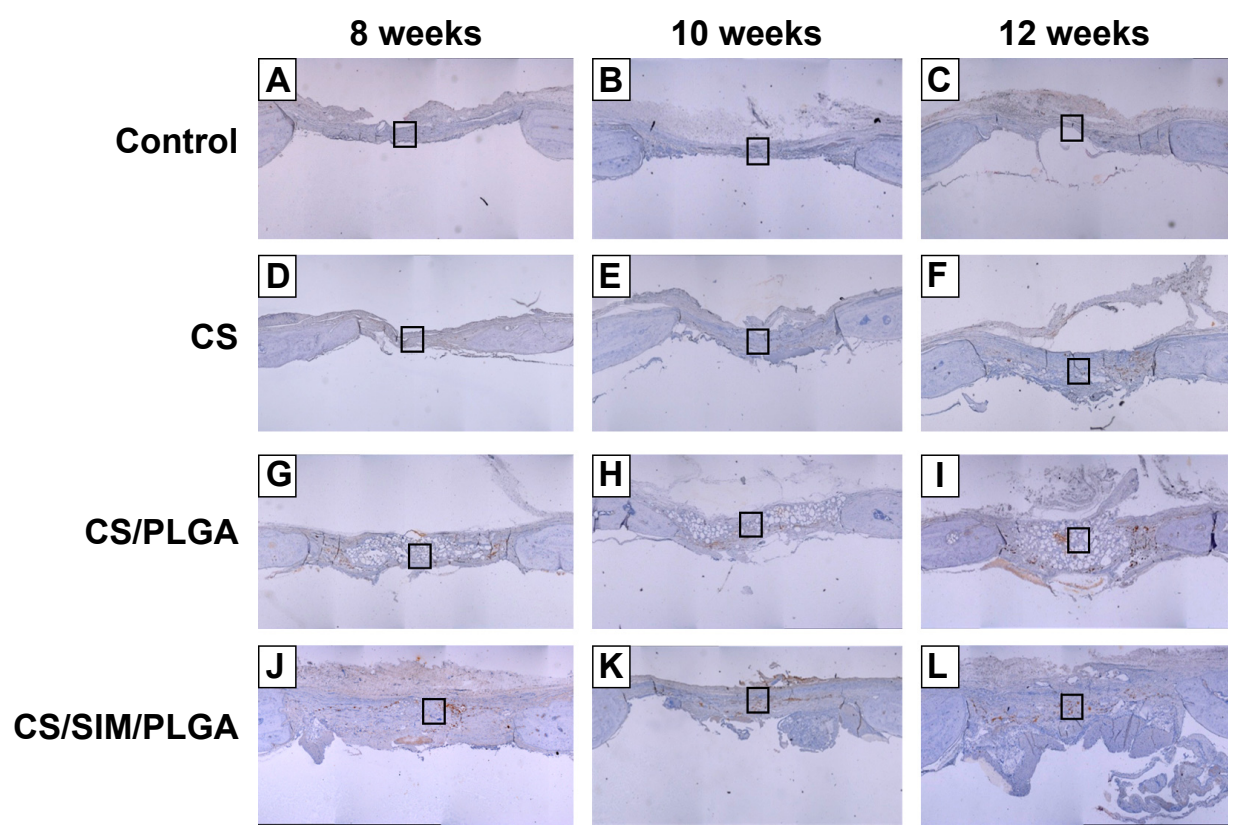

Figure 7 Immunohistochemistry analysis of bone morphogenetic protein-2 expression in calvarial defects 8, 10, and I2 weeks after implantation of bone graft substitutes. Notes: Calvarial defects implanted without bone graft substitutes were used as controls. (A-L) Higher magnification of the rectangle inset areas are shown as Figure 8. Abbreviations: CS, calcium sulfate; SIM, simvastatin; PLGA, poly(lactic-co-glycolic acid) microspheres.

A meta-analysis of studies comparing $\mathrm{CP}$ bone substitutes with autografts suggested that use of $\mathrm{CP}$ bone substitutes circumvented the need for a second incision to harvest autografts, was associated with fracture reduction, and likely improved functional outcomes. ${ }^{32,33}$ Larsson et al have reported the effect of these substitutes in fractures of the tibia plateau, hip, and distal radius. ${ }^{33}$ However, according to a study by Russell et al approximately $10 \%$ of the $\mathrm{CP}$ was

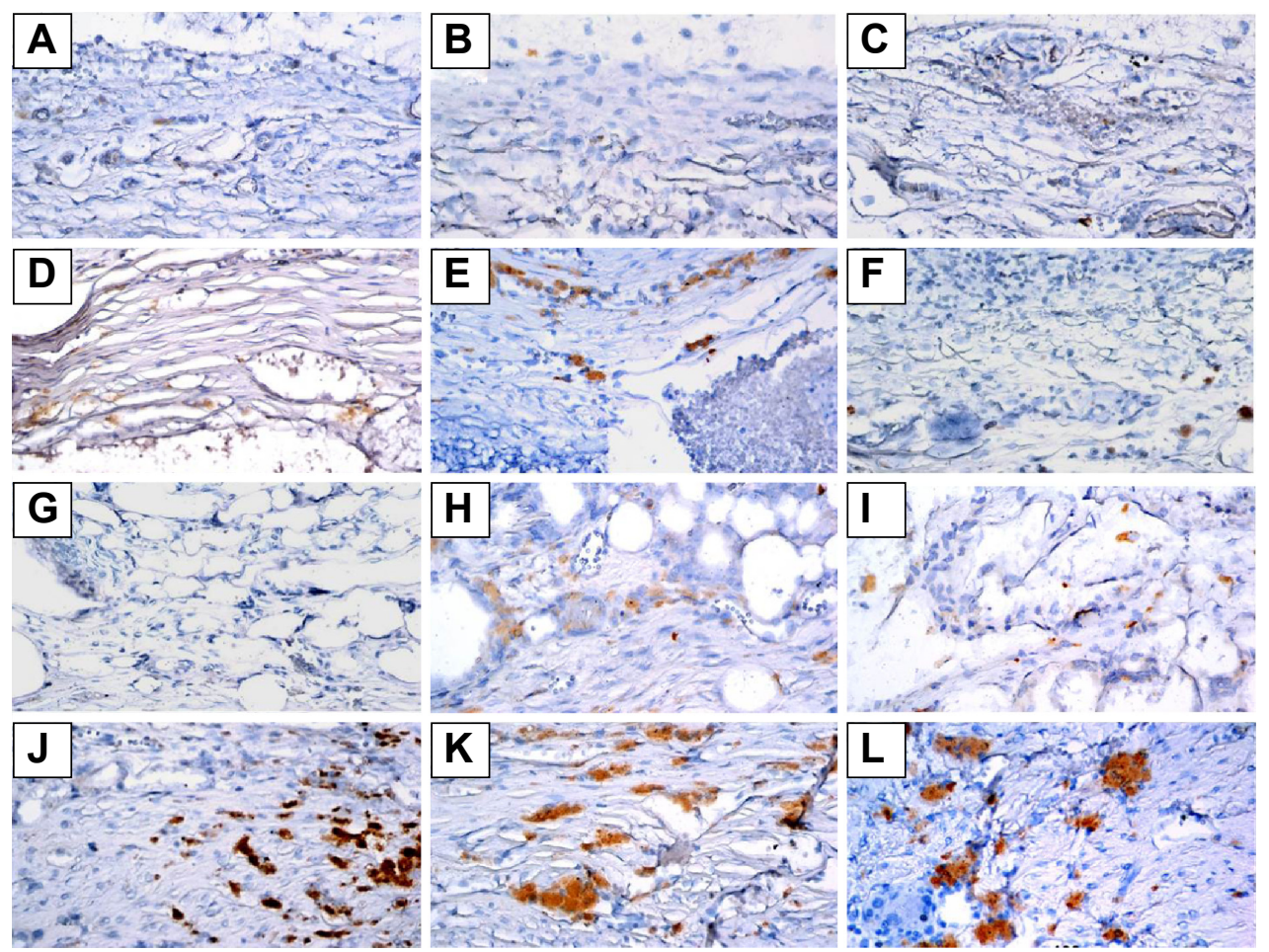

Figure 8 Immunohistochemistry analysis of bone morphogenetic protein-2 expression in calvarial defects at 8, I0, and I2 weeks after implantation of bone graft substitutes. Notes: Calvarial defects implanted without bone graft substitutes were used as controls. A-L show the higher magnification of the rectangle inset areas in Figure 7. Control group (A-C); CS group (D-F); CS/PLGA group (G-I); CS/SIM/PLGA group (J-L). 


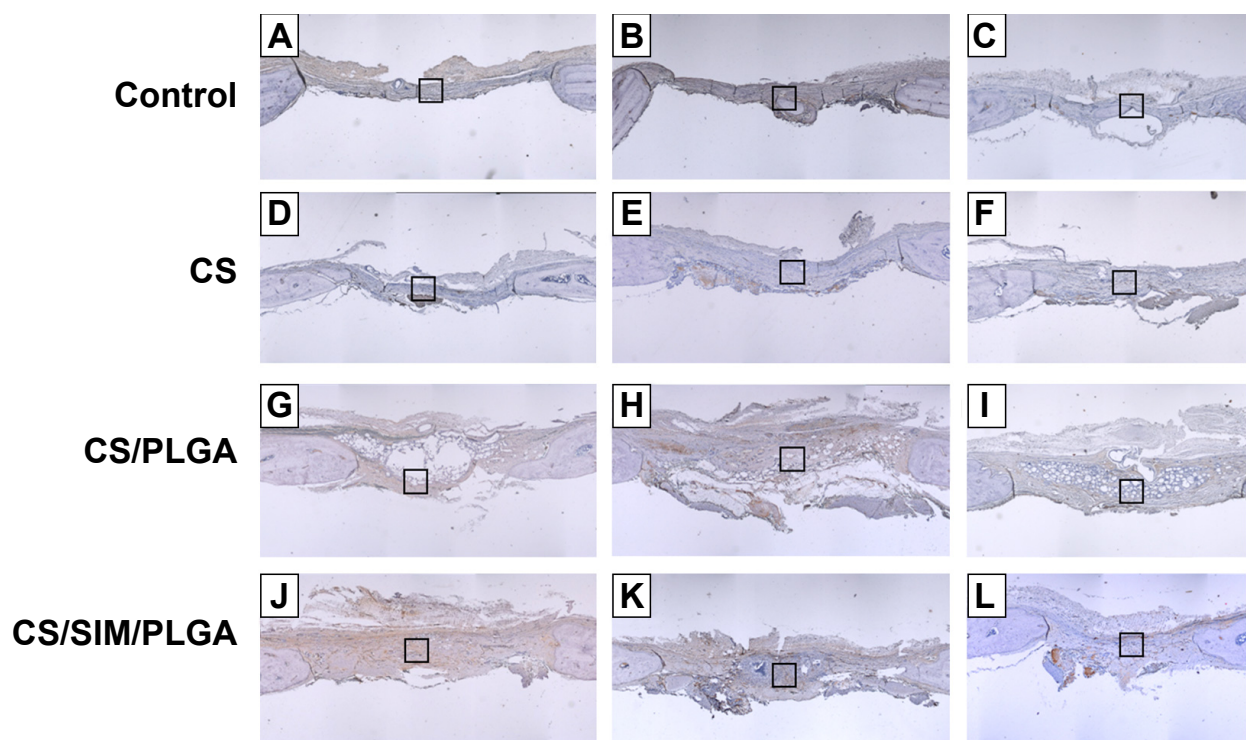

Figure 9 Immunochemistry analysis of von Willebrand factor expression in calvarial defects at 8, I0, and I2 weeks after implantation of bone graft substitutes. Notes: Calvarial defects implanted without bone graft substitutes were used as controls. (A-L) Higher magnification of the rectangle inset areas are shown as Figure 10. Abbreviations: CS, calcium sulfate; SIM, simvastatin; PLGA, poly(lactic-co-glycolic acid) microspheres.

still visible in most patients at one year after implantation surgery. ${ }^{34}$ The use of similar substances such as CS has not been as successful as using CP. Ziran et al reported that use of CS in non-unions led to no improvement in bone healing and increased wound drainage because of its rapid resorption within 6-8 weeks. ${ }^{35}$ Therefore, CS is not recommended for non-unions or periarticular fractures, but Osteoset bone graft substitutes may be used as a drug carrier to deposit tobramycin in local areas. According to the aforementioned studies, CP is difficult to resorb within one year in clinical use, and HAp lasts longer than CP. By contrast, CS is easily resorbed but has drug deposit properties.

Flierl et al reported that the surgical management of long bone fracture non-unions with allografts had higher
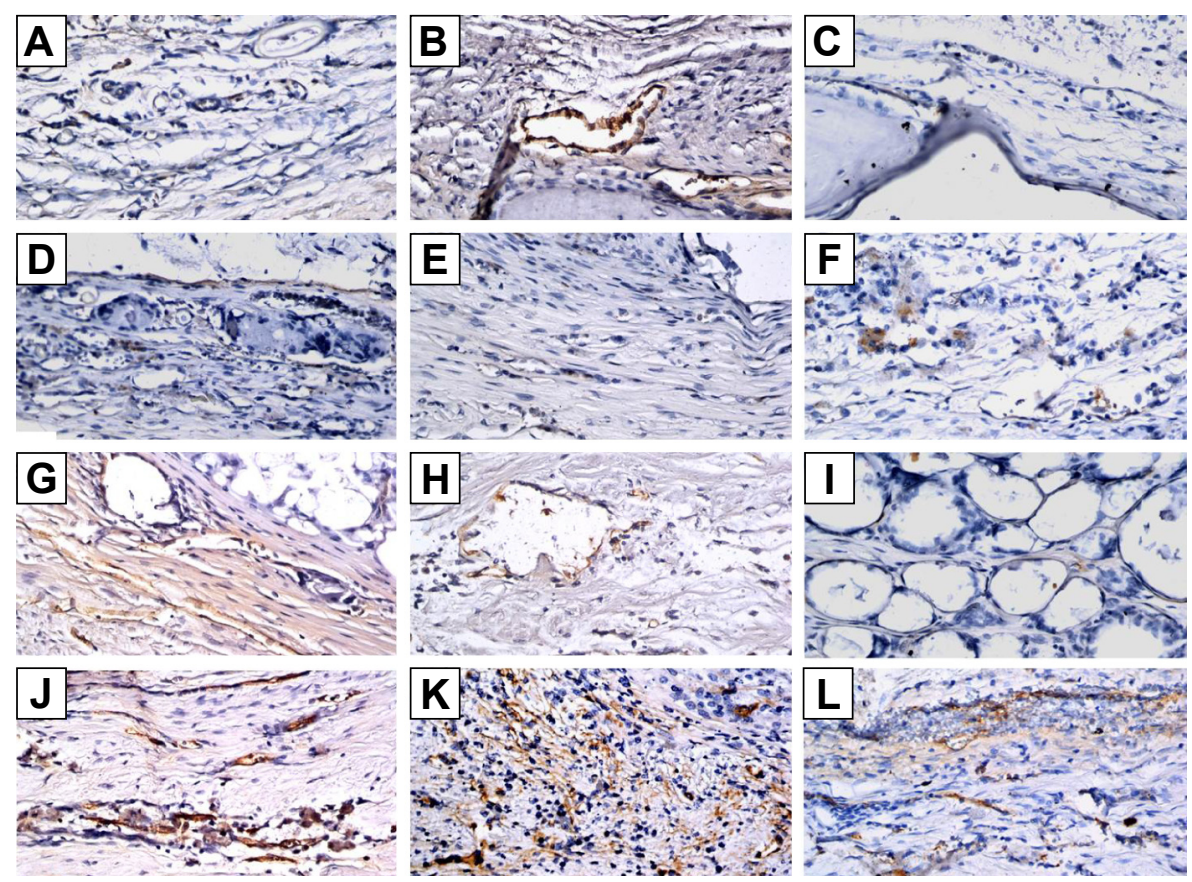

Figure 10 Immunohistochemistry analysis of von Willebrand factor expression in calvarial defects at 8, 10 , and I2 weeks after implantation of bone graft substitutes. Notes: Calvarial defects implanted without bone graft substitutes were used as controls (magnification $400 \times$ ). A-L show the higher magnification of the rectangle inset areas in Figure 9. Control group (A-C); CS group (D-F); CS/PLGA group (G-I); CS/SIM/PLGA group (J-L). 
rates of surgical revisions (47\%) and revision bone grafting $(32 \%) .{ }^{36}$ Our in vivo results show that the osteoconductive factor alone was not sufficient in healing critical-sized calvarial defects. We attribute this to the lack of osteoinductive factors stimulating neovascularization. Several in vitro studies have indicated that statins increase the expression of osteogenic marker genes other than BMP-2, including Runt-related transcription factor 2, osteocalcin, osteopontin, and alkaline phosphatase, in osteoblastic cells and bone marrow-derived cells. ${ }^{37-39}$ Therefore, SIM/PLGA can serve as an osteoinductive factor and improve the bone healing of critical-sized calvarial defects. In this study, BMP-2 staining showed that treatment with CS/SIM/PLGA continuously stimulated BMP-2 expression during the healing of criticalsized calvarial defects. BMP is known not only for being an osteoinductive agent that can enhance bone repair but also for being a potent stimulator of new blood vessel formation. ${ }^{40}$ This indicates that this novel CS/SIM/PLGA combination could provide an appropriate niche over the critical-sized defect area for bone repair. The vWF staining data showed that treatment with CS/SIM/PLGA promoted neovascularization at an early stage of bone healing and maintained this activity during the healing of critical-sized calvarial defects. This suggests that simvastatin-stimulated neovascularization might help transport stem cells into non-union areas to facilitate bone regeneration.

\section{Conclusion}

In this study, we demonstrated that a rapidly resorbable CS bone substitute with drug-carrying ability combined with osteoinductive SIM/PLGA substantially enhanced osteogenic and angiogenic activity and promoted bone healing in critical-sized calvarial defects in rats. We developed an ideal bone substitute, ie, CS/SIM/PLGA, which exhibited both osteoconduction and osteoinduction effects for clinical application. Our data suggest that this novel bone substitute combination may improve the biomechanical support for early callus formation after implanting the CS bone substitute, and thus may be used to heal substantial bone defects.

\section{Acknowledgments}

The authors gratefully acknowledge the support of research grants from the National Science Council and Kaohsiung Medical University Hospital and Kaohsiung Medical University Aim for the Top Universities Grant under the following grant numbers (NSC 99-2628-B-037-002-, KMUH1011R39, KMUH100-0R40, KMU-TP103B04).

\section{Author contributions}

All authors made substantial contributions to conception and design, acquisition of data, or analysis and interpretation of data; took part in either drafting the article or revising it critically for important intellectual content; gave final approval of the version to be published; and agree to be accountable for all aspects of the work.

\section{Disclosure}

The authors report no conflicts of interest in this work.

\section{References}

1. Calori GM, Albisetti W, Agus A, Iori S, Tagliabue L. Risk factors contributing to fracture non-unions. Injury. 2007;38 Suppl 2:S11-S18.

2. Einhorn TA. Clinically applied models of bone regeneration in tissue engineering research. Clin Orthop Relat Res. 1999;367 Suppl:S59-S67.

3. Tzioupis C, Giannoudis PV. Prevalence of long-bone non-unions. Injury. 2007;38 Suppl 2:S3-S9.

4. Aspenberg P, Genant HK, Johansson T, et al. Teriparatide for acceleration of fracture repair in humans: a prospective, randomized, doubleblind study of 102 postmenopausal women with distal radial fractures. J Bone Miner Res. 2010;25(2):404-414.

5. Lewandrowski KU, Gresser JD, Wise DL, Trantol DJ. Bioresorbable bone graft substitutes of different osteoconductivities: a histologic evaluation of osteointegration of poly(propylene glycol-co-fumaric acid)based cement implants in rats. Biomaterials. 2000;21(8):757-764.

6. Muschler GF, Negami S, Hyodo A, Gaisser D, Easley K, Kambic H. Evaluation of collagen ceramic composite graft materials in a spinal fusion model. Clin Orthop Relat Res. 1996;328:250-260.

7. Arrington ED, Smith WJ, Chambers HG, Bucknell AL, Davino NA. Complications of iliac crest bone graft harvesting. Clin Orthop Relat Res. 1996;329:300-309.

8. Summers BN, Eisenstein SM. Donor site pain from the ilium. A complication of lumbar spine fusion. J Bone Joint Surg Br. 1989;71(4): 677-680.

9. Younger EM, Chapman MW. Morbidity at bone graft donor sites. J Orthop Trauma. 1989;3(3):192-195.

10. Beuerlein MJ, McKee MD. Calcium sulfates: what is the evidence? J Orthop Trauma. 2010;24 Suppl 1:S46-S51.

11. Damien CJ, Parsons JR. Bone graft and bone graft substitutes: a review of current technology and applications. J Appl Biomater. 1991;2(3):187-208.

12. Pietrzak WS, Ronk R. Calcium sulfate bone void filler: a review and a look ahead. J Craniofac Surg. 2000;11(4):327-333.

13. Thomas MV, Puleo DA. Calcium sulfate: properties and clinical applications. J Biomed Mater Res B Appl Biomater. 2009;88(2):597-610.

14. Thomas MV, Puleo DA, Al-Sabbagh M. Calcium sulfate: a review. $J$ Long Term Eff Med Implants. 2005;15(6):599-607.

15. McAndrew MP, Gorman PW, Lange TA. Tricalcium phosphate as a bone graft substitute in trauma: preliminary report. J Orthop Trauma. 1988;2(4):333-339.

16. Schnurer SM, Gopp U, Kuhn KD, et al. [Bone substitutes]. Orthopade. 2003;32(1):2-10. German.

17. Zhou J, Gao C, Feng P, Xiao T, Shuai C, Penget S. Calcium sulfate bone scaffolds with controllable porous structure by selective laser sintering. J Porous Mater. 2015:1-8. Available from: http://link.springer.com/ article/10.1007/s10934-015-9993-x\#page-1. Accessed July 26, 2015.

18. Kamitakahara M, Ohtsuki C, Miyazaki T. Review paper: behavior of ceramic biomaterials derived from tricalcium phosphate in physiological condition. J Biomater Appl. 2008;23(3):197-212.

19. Lu J, Descamps M, Dejou J, et al. The biodegradation mechanism of calcium phosphate biomaterials in bone. J Biomed Mater Res. 2002; 63(4):408-412. 
20. Handschel J, Wiesmann HP, Stratmann U, et al. TCP is hardly resorbed and not osteoconductive in a non-loading calvarial model. Biomaterials. 2002;23(7):1689-1695.

21. Passuti N, Daculsi G, Rogez JM, Martin S, Bainvel JV. Macroporous calcium phosphate ceramic performance in human spine fusion. Clin Orthop Relat Res. 1989;248:169-176.

22. De Long WG Jr, Einhorn TA, Koval K, et al. Bone grafts and bone graft substitutes in orthopaedic trauma surgery. A critical analysis. J Bone Joint Surg Am. 2007;89(3):649-658.

23. Fischer J, Kolk A, Wolfart S, et al. Future of local bone regeneration - protein versus gene therapy. J Craniomaxillofac Surg. 2011;39(1):54-64.

24. Bennett M, Reynolds AS, Dickerman RD. Recent article by Shields et al titled "adverse effects associated with high-dose recombinant human bone morphogenetic protein-2 use in anterior cervical spine fusion”. Spine. 2006;31(17):2029-2030.

25. Perri B, Cooper M, Lauryssen C, Anand N. Adverse swelling associated with use of rh-BMP-2 in anterior cervical discectomy and fusion: a case study. Spine J. 2007;7(2):235-239.

26. Smucker JD, Rhee JM, Singh K, Yoon ST, Heller JG. Increased swelling complications associated with off-label usage of rhBMP-2 in the anterior cervical spine. Spine. 2006;31(24):2813-2819.

27. Corsini A, Bellosta S, Baetta R, Fumagalli R, Paoletti R, Bernini F. New insights into the pharmacodynamic and pharmacokinetic properties of statins. Pharmacol Ther. 1999;84(3):413-428.

28. Schachter M. Chemical, pharmacokinetic and pharmacodynamic properties of statins: an update. Fundam Clin Pharmacol. 2005; 19(1):117-125.

29. Mundy G, Garrett R, Harris S, et al. Stimulation of bone formation in vitro and in rodents by statins. Science. 1999;286(5446):1946-1949.

30. Tai IC, Fu YC, Wang CK, Chang JK, Ho ML. Local delivery of controlled-release simvastatin/PLGA/HAp microspheres enhances bone repair. Int J Nanomedicine. 2013;8:3895-3904.

31. Wang CZ, Chen SM, Chen CH, et al. The effect of the local delivery of alendronate on human adipose-derived stem cell-based bone regeneration. Biomaterials. 2010;31(33):8674-8683.
32. Doty HA, Leedy MR, Courtney HS, Haggard WO, Bumgardner JD. Composite chitosan and calcium sulfate scaffold for dual delivery of vancomycin and recombinant human bone morphogenetic protein-2. J Mater Sci Mater Med. 2014;25(6):1449-1459.

33. Larsson S, Hannink G. Injectable bone-graft substitutes: current products, their characteristics and indications, and new developments. Injury. 2011;42 Suppl 2:S30-S34.

34. Russell TA, Leighton RK; Alpha-BSM Tibial Plateau Fracture Study Group. Comparison of autogenous bone graft and endothermic calcium phosphate cement for defect augmentation in tibial plateau fractures. A multicenter, prospective, randomized study. J Bone Joint Surg Am. 2008;90(10):2057-2061.

35. Ziran BH, Smith WR, Morgan SJ. Use of calcium-based demineralized bone matrix/allograft for nonunions and posttraumatic reconstruction of the appendicular skeleton: preliminary results and complications. J Trauma. 2007;63(6):1324-1328.

36. Flierl MA, Smith WR, Mauffrey C, et al. Outcomes and complication rates of different bone grafting modalities in long bone fracture nonunions: a retrospective cohort study in 182 patients. J Orthop Surg Res. 2013;8:33.

37. Maeda T, Matsunuma A, Kawane T, Horiuchi N. Simvastatin promotes osteoblast differentiation and mineralization in MC3T3-E1 cells. Biochem Biophys Res Commun. 2001;280(3):874-877.

38. Song C, Guo Z, Ma Q, et al. Simvastatin induces osteoblastic differentiation and inhibits adipocytic differentiation in mouse bone marrow stromal cells. Biochem Biophys Res Commun. 2003;308(3):458-462.

39. Baek KH, Lee WY, Oh KW, et al. The effect of simvastatin on the proliferation and differentiation of human bone marrow stromal cells. J Korean Med Sci. 2005;20(3):438-444.

40. Deckers MM, van Bezooijen RL, van der Horst G, et al. Bone morphogenetic proteins stimulate angiogenesis through osteoblastderived vascular endothelial growth factor A. Endocrinology. 2002; 143(4):1545-1553.
International Journal of Nanomedicine

\section{Publish your work in this journal}

The International Journal of Nanomedicine is an international, peerreviewed journal focusing on the application of nanotechnology in diagnostics, therapeutics, and drug delivery systems throughout the biomedical field. This journal is indexed on PubMed Central, MedLine, CAS, SciSearch $®$, Current Contents ${ }^{\circledR} /$ Clinical Medicine,

\section{Dovepress}

Journal Citation Reports/Science Edition, EMBase, Scopus and the Elsevier Bibliographic databases. The manuscript management system is completely online and includes a very quick and fair peer-review system, which is all easy to use. Visit http://www.dovepress.com/ testimonials.php to read real quotes from published authors. 